\title{
Food security status in southern Nepal: application of the Household Food Insecurity Access Scale questions
}

\author{
D. Kular ${ }^{1}$, D. Devakumar ${ }^{1}$, D. S. Manandhar ${ }^{2}$, B. P. Shrestha ${ }^{2}$, N. M. Saville ${ }^{1}$ and D. Osrin ${ }^{1}$ \\ ${ }^{1}$ Institute for Global Health, University College London, London WC1N 1EH, UK and ${ }^{2}$ Mother and Infant Research \\ Activities, GPO Box 921, Kathmandu, Nepal
}

Food security exists when people have access at all times to adequate, safe and nutritious food for a healthy and active life. Food insecurity contributes to poor health and nutritional outcomes ${ }^{(1)}$. We assessed food security status in a cohort of households in Nepal's central Terai.

Data were collected in Dhanusha district. Food insecurity was assessed with the Household Food Insecurity Access Scale

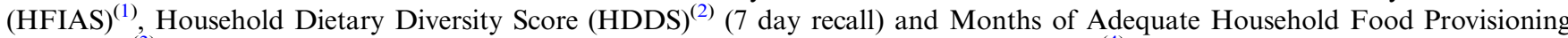
$(\text { MAHFP) })^{(3)}$. Socioeconomic status (SES) was determined by principal components analysis ${ }^{(4)}$. Chi-squared tests were used to compare urban and rural households, and univariable regression models to assess associations between socioeconomic status and food security measures.

In 837 households (340 urban), mean HFIAS score was 0.69 (SD 2.80) and the prevalence of food insecurity was $8.6 \%(n=72)$. There was no difference between urban and rural households. Mean HDDS was 9.24, and higher in urban households. $92 \%$ of respondents had no difficulty obtaining food in the past 12 months; $3 \%$ reported difficulty obtaining food for 3 months or longer. There was no difference between rural and urban locations except in the post-monsoon season, when rural households had greater food insecurity. A test for trend showed an inverse relationship between socioeconomic quintile and HFIAS score $(p<0.001)$. A positive association was seen between socioeconomic quintile and consumption of meat, dairy or eggs. Our data suggest a greater proportion of food secure households (50\% vs 92\%) than described in the Nepal Demographic and Health Survey (NDHS) for the central Terai ${ }^{(5)}$.

Most households in our cohort were food secure all year round and had a diverse dietary intake, considerably better than that shown in the NDHS. The likelihood of being food secure was related to SES and rural residents were more vulnerable than urban residents in the post-monsoon period.

1. Coates J, Swindale A \& Bilinsky P (2007) Household Food Insecurity Access Scale (HFIAS) for Measurement of Food Access: Indicator Guide.

2. Swindale A \& Bilinsky P (2006) Household Dietary Diversity Score (HDDS) for Measurement of Household Food Access: Indicator Guide.

3. Bilinsky P \& Swindale A (2007) Months of Adequate Household Food Provisioning (MAHFP) for Measurement of Household Food Access: Indicator Guide.

4. Vyas S \& Kumaranayake L (2006) Constructing socio-economic status indices:how to use principal components analysis. Health Policy Plan, 21, $459-468$.

5. Ministry of Health and Population (MOHP) (2012) [Nepal], New ERA, and ICF International. Nepal Demographic and Health Survey 2011. Kathmandu, Nepal: Ministry of Health and Population, New ERA, and ICF International. 